\title{
Degradación de hojarasca y aporte de nutrientes del manglar en la Laguna Mecoacán, Golfo de México
}

Jony R. Torres V. ${ }^{1}$, Dulce Infante-Mata ${ }^{2}$, Alberto J. Sánchez ${ }^{3}$, Alejandro Espinoza-Tenorio ${ }^{1}$ \& Everardo Barba ${ }^{1 *}$

1. Manejo sustentable de cuencas y zonas costeras. El Colegio de la Frontera Sur. Carretera a Reforma, km $15.5 \mathrm{~s} / \mathrm{n}$ Ra. Guineo 2da Sección. C.P. 86280 Villahermosa, Tabasco, México; jonyc97@hotmail.com, aespinoza@ecosur.mx, ebarba@ecosur.mx*

2. Manejo sustentable de cuencas y zonas costeras. El Colegio de la Frontera Sur. Unidad Tapachula; dulce.infante@gmail.com

3. Laboratorio Manejo de Humedales Tropicales. DACBIOL, Universidad Juárez Autónoma de Tabasco; alberthoj.sanchez@gmail.com

* Correspondencia

Recibido 06-IX-2017. Corregido 06-II-2018. Aceptado 07-III-2018.

\begin{abstract}
Leaf litter degradation and nutrient supply in the mangroves of Mecoacán Lagoon, Gulf of Mexico. Mangroves are ecosystems with a high primary productivity that is mainly driven mainly by degradation processes. Energy (nutrients) flows from mangroves toward adjacent zones and the surrounding aquatic environment. The objective of the present study was to estimate the daily degradation coefficient (k) of mangrove leaves in relation to physical-chemical soil factors and in situ nutrient supply (phosphorus and nitrogen) in Mecoacán Lagoon, Gulf of Mexico. Leaf litter degradation bags were placed at six monthly monitoring sites to evaluate degradation and to calculate the corresponding degradation coefficients. A rapid degradation of up to 51 $\%$ was observed for Avicennia germinans (L.) Stearn during the first month in association with leaching resulting from flood conditions. The degradation of Rhizophora mangle $(\mathrm{L}).(\mathrm{k}=0.0052 \pm 0.0002)(\mathrm{F}=12.2 \mathrm{p}<0.05 \mathrm{n}=$ 216) and Laguncularia racemosa (L.) Gaertn ( $\mathrm{k}=0.005 \pm 0.0003)(\mathrm{F}=3.7 \mathrm{p}=0.2 \mathrm{n}=108)$, differed significantly from that of $A$. germinans $(\mathrm{k}=0.009 \pm 0.0003)(\mathrm{F}=1.2 \mathrm{p}=0.2 \mathrm{n}=216)$ did not present significant differences. To reach $\mathrm{T}_{50}$ degradation, $R$. mangle and $L$. racemosa required more time (133 and 138 days, respectively) than A. germinans (74 days). Organic matter and soil humidity were significantly correlated with the decay constant of $A$. germinans ( $\mathrm{r}=0.65 \mathrm{p}<0.05$ and $\mathrm{r}=0.55 \mathrm{p}<0.05$, respectively). Total nitrogen content was highest in the Pajaral site $(2683 \mathrm{mg} . \mathrm{kg})$ and was also highly correlated with organic matter content $(\mathrm{r}=0.9 \mathrm{p}=0.003)$. Total phosphorus content was highest in the Boca site $(2031 \mathrm{mg} . \mathrm{kg})$ and was also negatively correlated with $\mathrm{pH}(\mathrm{r}=$ $-0.61 \mathrm{p}=0.004)$. In conclusion, differences in the rate of mangrove leaf degradation depend on the involved species (leaf composition), time of exposure or immersion in water (flooding patterns) and sediment heterogeneity (i.e., texture, pH, humidity content and bulk density). Rev. Biol. Trop. 66(2): 892-907. Epub 2018 June 01.
\end{abstract}

Key words: mangrove; degradation; litter; hydroperiod; leaching.

La alta productividad de los manglares a menudo se atribuye a las altas tasas de degradación de hojarasca y al reciclaje eficiente de nutrientes, tanto autóctonos como alóctonos, que provienen de fuentes naturales y antropogénicas (Lee, 1990; Bouillon, Koedam, Raman, \& Dehairs, 2002). Las diferencias en la velocidad de degradación de la hojarasca se pueden atribuir a la composición de especies del manglar, contenido de humedad y características físicas del suelo (Melillo, Naiman, Aber, \& Eshleman, 1983; Dick \& Osunkoya, 2000; Orihuela, Tovilla, Vester, \& Álvarez, 2004; Barroso-Matos, Bernini, \& Rezende, 2012), la morfología y composición de la hoja; por ejemplo la concentración de taninos inhibe la acción microbiana y de otros consumidores (Ashton, Hogarth, \& Ormond, 1999; Neilson, Giddins, 
\& Richards, 1986; Steinke, Barnabas, \& Samuru, 1990). Los suelos más finos (arcillas) tienen la capacidad de absorber y acumular materia orgánica, diferentes formas de nutrientes (Bailey, 1968) e influye en la dinámica del agua derivado de la porosidad y permeabilidad del suelo (Bjorn \& McClaugherty, 2008). La inundación en humedales tiene efectos sobre la descomposición y el ciclo de nutrientes (Gerritsen \& Greening, 1989; OldeVenterink, Pieterse, Belgers, Wassen, \& de-Ruiter, 2002), permitiendo que estén disponibles para el nuevo crecimiento y desarrollo de las plantas (Chapman, 1986; Shunula \& Whittick, 2001). La degradación de la materia orgánica en manglar asegura que una gran parte de la producción de materia orgánica por hojarasca se recicle dentro del bosque influido por el tipo de bosque de manglar (riberino, cuenca, borde) que reducen las exportaciones por mareas hacia ecosistemas acuáticos adyacentes (Lugo \& Snedaker, 1974; Twilley, Lugo, \& Patterson-Zucca, 1986; Hemminga et al., 1994; Lee, 1998; Tovilla, 1994) ha informado que hasta $40 \%$ de la materia orgánica producida por los ecosistemas costeros mexicanos, es transportada al mar y áreas adyacentes a través de la hojarasca. Relacionado con la productividad y degradación de hojarasca se puede citar a Sessegolo y Lana, 1991; Twilley et al., 1997; Juman, 2005; Middleton y Mckee, 2001; en México los trabajos realizados por Aké-Castillo, Vázquez y López-Portillo (2006) en la laguna Sontecomapan, Veracruz y Orihuela et al., (2004) en el sistema lagunar de Pampa, Chiapas. El presente trabajo tiene como objetivos (1) estimar el coeficiente de degradación diario (k) de las hojas de tres especies de mangle en relación con los factores físico químicos del suelo y (2) determinar el contenido de nutrientes (fósforo y nitrógeno) en sedimento del manglar en la Laguna Mecoacán. Para lograr estos objetivos se plantearon las tres siguientes hipótesis: (1) La hoja de $A$. germinans registrará la degradación más rápida; mientras que la degradación de $R$. mangle y L. racemosa será más lenta, por las diferencias en la composición de la hoja; (2) el coeficiente de degradación diario $(\mathrm{k})$ tendrá alta correlación positiva con el tipo de suelo arcilloso con mayor capacidad de retención de humedad que propicia la lixiviación de la hoja de mangle; y (3) el contenido de materia orgánica fósforo y nitrógeno en suelo del manglar estará directamente correlacionado con el coeficiente de degradación de la hoja de mangle $(\mathrm{k})$.

\section{MATERIALES Y MÉTODOS}

Área de estudio: La Laguna Mecoacán tiene una extensión de 96454.39 ha (Barba, Rangel, \& Ramos, 2006) y se ubica en el sur del Golfo de México (18 $16^{\circ}$ - $18^{\circ} 20^{\prime}$ N \& $\left.93^{\circ} 04^{\prime}-93^{\circ} 14^{\prime} \mathrm{W}\right)$. En el margen este y sureste de la misma desembocan los ríos Escarbado y Cuxcuchapa (Gómez, 1977) (Fig. 1). La salinidad fluctúa entre 0.5 y 29 ups; los valores mínimos se registran al sureste del sistema en temporada de precipitación y las máximas salinidades al norte y oeste en temporada de sequía (Domínguez, Sánchez, Florido, \& Barba, 2003). La vegetación dominante en la laguna son Rhizophora mangle L. (mangle rojo), Laguncularia racemosa (L.) Gaertn (mangle blanco) y Avicennia germinans (L.) Stearn (mangle negro, madresal) (Pennington \& Sarukhán, 2005).

Sitios de muestreo: Se seleccionaron seis sitios (Fig. 1) en zonas conservadas de manglar (Domínguez-Domínguez, Zavala-Cruz, \& Martínez-Zurimendi, 2011), al norte de la laguna se ubicó el sitio Boca que se encuentra directamente influenciado por corrientes de marea y Cerros como zona de mezcla en el flujo de entrada y salida de la corriente de la laguna (Gómez, 1977), hacia el este se establecieron los sitios Pajaral y Arrastradero con mayor influencia de los ríos Cuxcuchapa y Escarbado (Hernández, 2007), hacia el suroeste y oeste de la laguna se ubicaron los sitios Aspoquero y Mojarrero con menor aporte de agua dulce y mayor salinidad (Domínguez et al., 2003; Infante-Mata et al., 2014). En cada sitio se instalaron 3 unidades de muestreo (UM) de $10 \times 10 \mathrm{~m}$, a una distancia de $20 \mathrm{~m}$ de la línea 

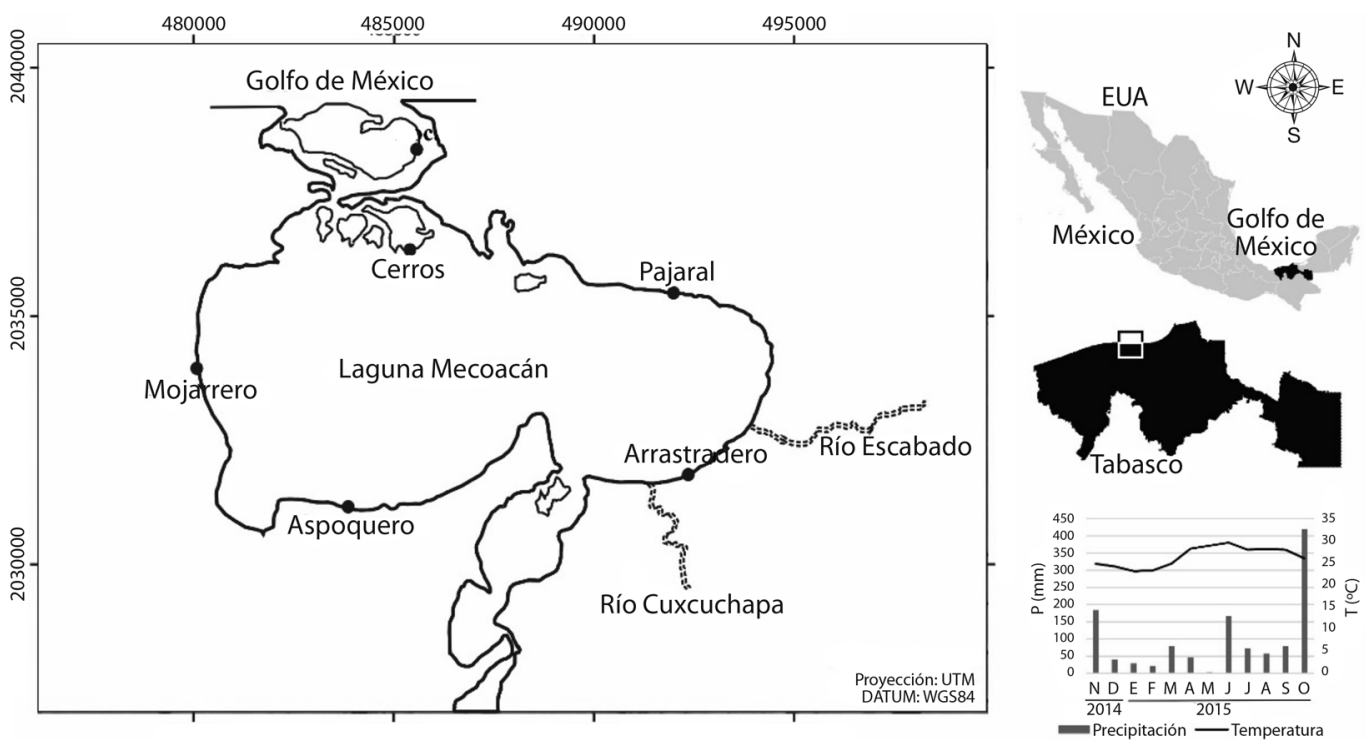

Fig. 1. Sitio de estudio, Laguna Mecoacán, Golfo de México. (*) Datos de precipitación tomados de la Torre meteorológica CONAGUA-SMN-EMAS (2015).

Fig. 1. Study site, Mecoacan Lagoon, Gulf of Mexico. (*) Precipitation data taken from the Meteorological Tower CONAGUA-SMN-EMAS (2015).

de costa con separación de $50 \mathrm{~m}$ entre cada UM. El período de estudio fue de octubre 2014 a noviembre 2015, durante las temporadas climáticas representativas de la región: nortes (noviembre a febrero), secas (marzo a junio) y lluvias (julio a octubre).

\section{Atributos físico químicos en sedimento:} Mensualmente se recolectó una muestra de sedimento en los primeros $20 \mathrm{~cm}$ en cada UM (216 muestras) mediante un nucleador $(0.0033$ $\mathrm{m}^{2}$ ) para su posterior análisis de nitrógeno total (NT) y fósforo (PT) total de acuerdo a la NOM-021-SEMARNAT-2000, así como la medición de textura por método de Boyucos (Klute, 1986), pH en relación 1:2 en agua por electrometría, materia orgánica (Walkley \& Black, 1934), la densidad aparente y el contenido de humedad del suelo se midieron de acuerdo a lo propuesto por Moreno-Casasola y Warner (2009), el contenido de humedad del suelo es el peso de agua que es capaz de almacenar un gramo de suelo, si el valor fuera $100 \%$ significaría que $1 \mathrm{~g}$ de suelo almacena $1 \mathrm{~g}$ de agua (Infante-Mata, 2011).
Hidroperíodo: En cada UM se instaló un piezómetro (18 en total) con $1.5 \mathrm{~m}$ de profundidad donde se midió mensualmente el nivel de inundación de acuerdo a lo propuesto por Peralta, Infante-Mata y Moreno-Casasola, (2009). Los piezómetros se construyeron con tubos de PVC de 1 pulgada de diámetro y 3 $\mathrm{m}$ de alto $(1.5 \mathrm{~m}$ del tubo queda por debajo del nivel del suelo), en $20 \mathrm{~cm}$ del extremo que quedó enterrado se hicieron ranuras alternas cada $2 \mathrm{~cm}$ y se cubrieron con malla de 250 micras, la cual quedó inmovilizada con alambre inoxidable.

Degradación de hoja de mangle: Se utilizó el método de bolsas de degradación propuesto por Bosire et al., (2005). En cada UM se instalaron sobre la superficie del suelo 12 bolsas de degradación de nylon de $15 \times 20 \mathrm{~cm}$ de $1 \mathrm{~mm}^{2}$ de malla, con 20 gramos en peso seco de hoja de mangle de cada especie ( $R$. mangle, $A$. germinans y L. racemosa) y hojas combinadas, que corresponde a la mezcla de hojas de las tres especies de mangle en proporciones al azar para replicar las condiciones naturales de 
la hoja en suelo del bosque de manglar. La hoja senescente se colectó directamente del árbol, la cual se secó durante $90 \mathrm{hrs} \mathrm{a} 60{ }^{\circ} \mathrm{C}$ (Orihuela et al., 2004). Las bolsas de degradación de $L$. racemosa sólo se instalaron en los sitios en donde estaba presente (Cerros, Mojarrero y Arrastradero) (total 756 litterbags). Una bolsa de degradación de cada especie y otra de hojas combinadas se colectó mensualmente en cada UM (4 bolsas de degradación por tratamiento) (63 total). Todas las bolsas se llevaron al laboratorio en donde se lavaron cuidadosamente para remover los restos de sedimento y materia orgánica exterior a la bolsa, la hoja residual se secó a peso constante para obtener el porcentaje de pérdida de biomasa inicial, equivalente a la degradación. La tasa de degradación (R) se calculó de acuerdo a los propuesto por Juman (2005) restando el peso final (FW) del peso inicial (WI) y dividido por el número de días transcurridos (D).

El coeficiente de degradación diario en hoja de mangle $(\mathrm{k})$, se calculó mediante la función exponencial propuesta por Harmon, Nadelhoffer y Blair, (1999) probada y utilizada previamente por otros estudios (Chale, 1993; Twilley et al., 1997; Aké-Castillo et al., 2006).

Donde $\mathrm{k}$ es el coeficiente de degradación en días, $\mathrm{t}=$ tiempo (días), masa remanente en gramos de t, masa inicial. Se estimó el tiempo requerido para la degradación de la mitad del material foliar inicial $\mathrm{T}_{50}\left(\mathrm{~T}_{50}=(\ln )(2) / \mathrm{k}\right)$. Se realizó un análisis de la tasa de degradación $(\mathrm{R})$ y del coeficiente de degradación ( $\mathrm{k}$ ) de la hoja de mangle por temporadas de la región: nortes (noviembre a febrero), secas (marzo a junio) y lluvias (julio a octubre).

Análisis de datos: Los datos fueron explorados mediante la prueba de normalidad Kolmogorov-Smirnov y homogeneidad de varianzas de Levene (Steel \& Torrie, 1996). Las diferencias en los grupos de datos ordenados espacial (sitios) y temporalmente (meses), se estimaron con un nivel de significancia del $5 \%$, mediante ANDEVA de una vía de Tukey. Se aplicó un análisis de medidas repetidas y de covarianza (ANCOVA) para determinar las diferencias entre el porcentaje de degradación mensual con respecto al tipo de hoja de mangle (especie). Las correlaciones espaciales entre variables de arena, limo y arcilla con el contenido de humedad, materia orgánica, nitrógeno y densidad aparente en suelo; así como las correlaciones del coeficiente de degradación (k) con el contenido de arcilla se determinaron mediante correlaciones de Pearson (r). Los análisis se realizaron con el software SPSS versión 20; así mismo, se aplicó un análisis de correlación ( $\mathrm{r}$ ) del contenido humedad con hidroperíodo y densidad aparente del suelo en los meses de monitoreo.

\section{RESULTADOS}

Atributos físico químicos en sedimento: Temporalmente solo el tipo de textura limo mostró diferencias significativas $(\mathrm{F}=2.3 \mathrm{p}=$ $0.01 \mathrm{n}=216$ ), las diferencias son los valores bajos registrados en diciembre $(29.9 \pm 2.9 \%)$ en relación a los altos contenidos de limo en junio $(42 \pm 2.8 \%)$ y julio $(43 \pm 1.9 \%)$. El tipo de textura promedio del sedimento en los sitios registró porcentajes de $33.5 \pm 0.8$ de arena, $38.8 \pm 0.7$ de limo y $27.6 \pm 0.8$ de arcilla. Se encontraron diferencias significativas espaciales en los porcentajes de tipo de textura del sedimento $(\mathrm{F}=49 \mathrm{p}<$ $0.05 n=216$ ), se registró en Cerros, Aspoquero, Boca y Arrastradero un mayor porcentaje de arena $(38,44,45$ y $47 \%$ respectivamente), Mojarrero presentó mayor porcentaje de limo (43\%), mientras que Pajaral registró mayor porcentaje de arcilla (40\%) (Fig. 2). La densidad aparente del suelo en los sitios presentó diferencias significativas, esto debido al bajo valor registrado en Cerros $\left(0.7 \mathrm{~g} . \mathrm{cm}^{3}\right)$ y el más

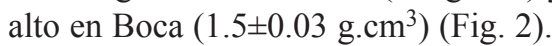

El pH presentó diferencias significativas, estas diferencias se presentaron por el bajo valor de $\mathrm{pH}$ en la Boca (3.7) en relación con los demás sitios (Fig. 2), temporalmente los valores de $\mathrm{pH}$ presentaron diferencias significativas $(\mathrm{F}=2.5 \mathrm{p}=0.01 \mathrm{n}=216)$, en un rango de $4.5 \pm 0.18$ en el mes de mayo hasta $5.7 \pm 0.17$ en octubre. El porcentaje de materia orgánica (MO) presentó diferencias significativas entre 

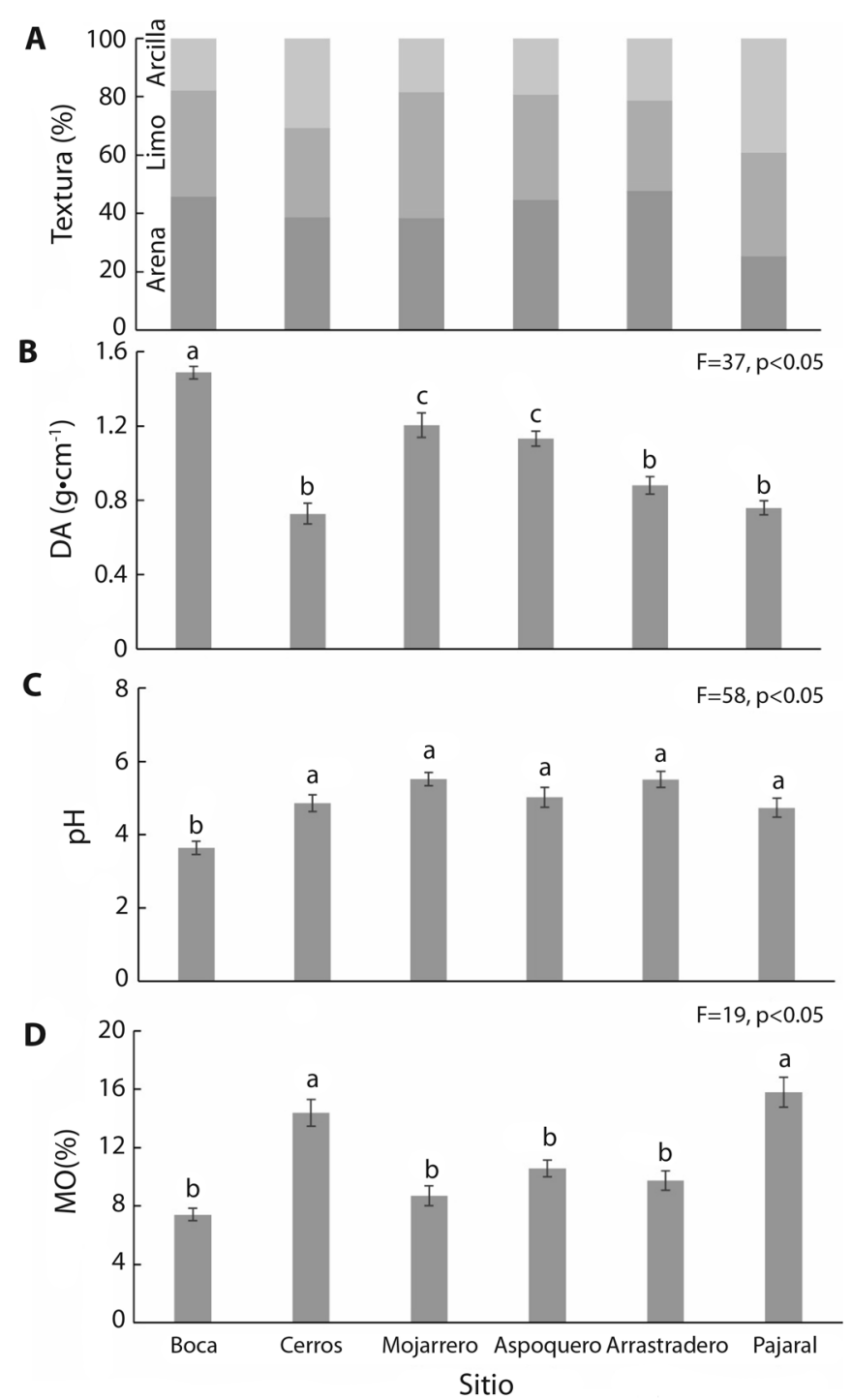

Fig. 2. Características físico-químicas del sedimento. (A) textura, (B) densidad aparente, (C) pH y (D) materia orgánica ( \pm ES). Letras muestran diferencias significativas entre sitios (Tukey $\mathrm{p}<0.05$ ).

Fig. 2. Physical and chemical characteristics of sediment. (A) texture, (B) bulk density, (C) pH and (D) organic matter ( \pm $\mathrm{SE}$ ). Letters show significant differences between sites (Tukey $\mathrm{p}<0.05$ ).

sitios, las diferencias se presentaron debido a los altos valores registrados en Cerros y Pajaral (14.5 \pm 0.9 y $15.8 \pm 1.1 \%$ respectivamente) en relación a los demás sitios, temporalmente el contenido de MO registró diferencias significativas $(\mathrm{F}=5.1 \mathrm{p}<0.05 \mathrm{n}=216)$ por la alta variación, con valor mínimo en diciembre (5.8 \%) y el máximo en agosto (14.1\%). (Fig. 2), además el contenido de $\mathrm{MO}$ presentó alta correlación negativa con el contenido arena $(\mathrm{r}=-0.79 \mathrm{p}=$ $0.04)$ y positiva con arcilla $(\mathrm{r}=0.94 \mathrm{p}=0.006)$.

El contenido de humedad en suelo durante el ciclo de estudio no presentó diferencias significativas $(F=1.3 p=0.2 n=216)$ con el mayor contenido de humedad en noviembre $(144 \pm 24 \%)$ y el menor en abril $(85 \pm 11 \%)$. 
Espacialmente el contenido de humedad presentó diferencias significativas $(\mathrm{F}=19.2 \mathrm{p}<$ $0.05 \mathrm{n}=216$ ), el mayor registro fue en Cerros (163 $\pm 14 \%$ ) y Pajaral (137 $\pm 11 \%)$ seguidos por Arrastradero (108 $\pm 10 \%$ ) y los sitios con menor almacenamiento de agua fueron Mojarrero (79 $\pm 11 \%)$ y Boca (51 $\pm 2 \%$ ) (Fig. 3), se identificó alta correlación positiva entre el contenido de humedad y el porcentaje de arcilla en suelo $(\mathrm{r}=0.82 \mathrm{p}=0.05)$ y correlación negativa con la densidad aparente $(r=-0.96 \mathrm{p}<0.05)$ del suelo.
Hidroperíodo: Ésta variable presentó diferencias significativas durante los meses de estudio $(\mathrm{F}=7.6 \mathrm{p}<0.05 \mathrm{n}=216)$, debido a una alta variación del patrón de inundación en la laguna con un máximo nivel en Cerros ( $19 \pm 0.5 \mathrm{~cm}$ en octubre) y el mínimo en Aspoquero ( $-122 \pm 12.5 \mathrm{~cm}$ en mayo). Todos los sitios se mantuvieron con inundación de octubre a diciembre (Fig. 3). Así mismo, se presentó alta correlación positiva del hidroperíodo y el contenido de humedad en suelo $(\mathrm{r}=0.8, \mathrm{p}=0.002)$ durante el ciclo de estudio.
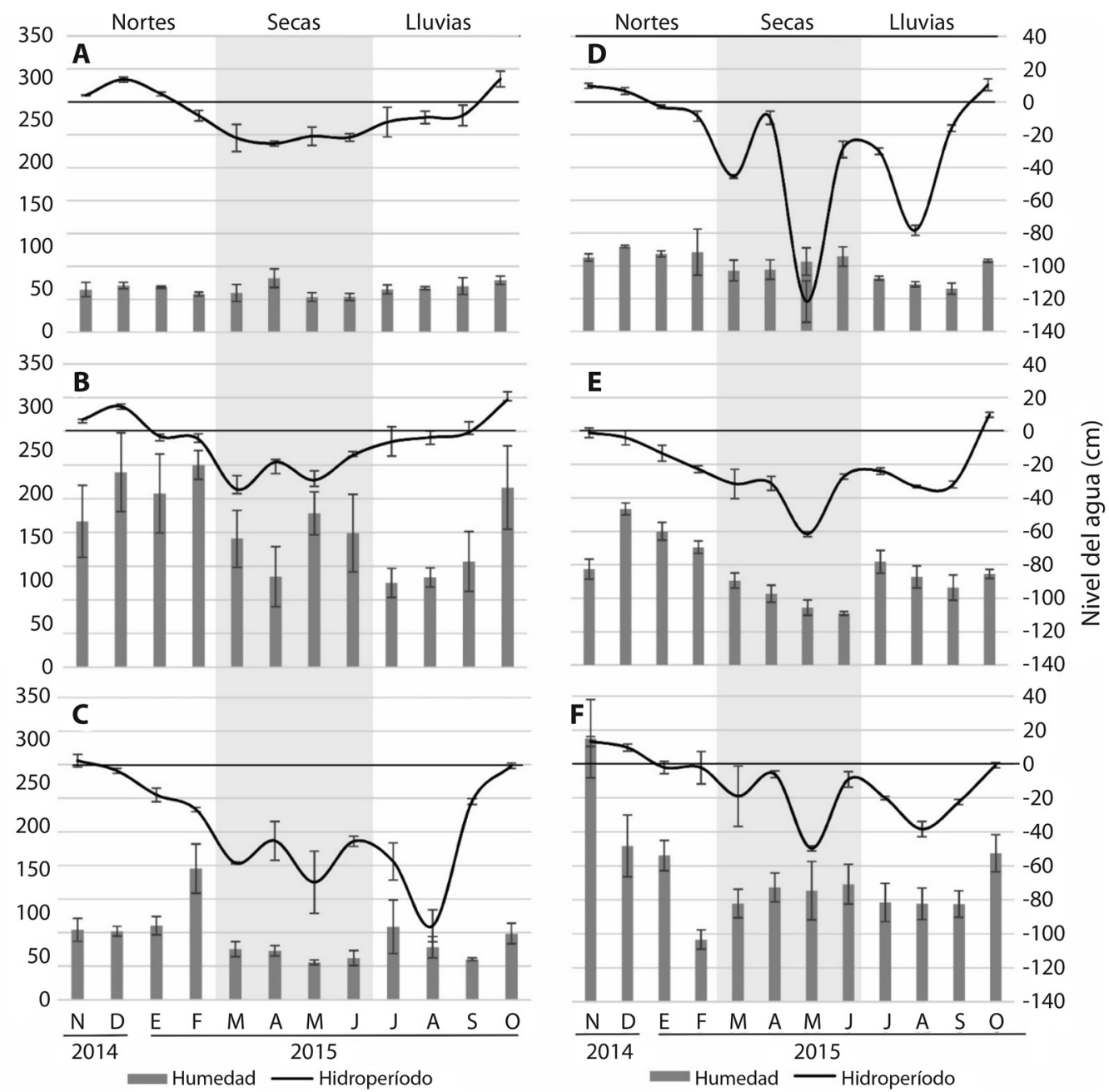

Fig. 3. Contenido de humedad en suelo e hidroperíodo mensual en cada sitio de estudio ( \pm ES). (A) Boca, (B) Cerros, (C) Mojarrero, (D) Aspoquero, (E) Arrastradero y (F) Pajaral.

Fig. 3. Humidity content in soil and monthly hydroperiod at each site ( \pm SE). (A) Boca, (B) Cerros, (C) Mojarrero, (D) Aspoquero, (E) Arrastradero and (F) Pajaral. 
Nutrientes (fósforo y nitrógeno) en suelo: Temporalmente el contenido de nitrógeno en suelo no presentó diferencias significativas $(\mathrm{F}=0.4 \mathrm{p}=0.95 \mathrm{n}=72)$ desde $0.21 \%$ (2067 mg.Kg) como máximo nivel en junio hasta el mínimo en diciembre con $0.12 \%$ (1217 mg.Kg). Entre sitios se presentaron diferencias significativas en contenido de nitrógeno $(\mathrm{F}=16.2 \mathrm{p}>0.05 \mathrm{n}=72)$, con alto contenido de nitrógeno en Pajaral $(0.27 \pm 0.02 \% ; 2683$ mg.Kg) y Cerros $(0.18 \pm 0.02 \% ; 1775 \mathrm{mg} . \mathrm{Kg})$ (Fig. 4). Se presentó una alta correlación positiva del nitrógeno con materia orgánica $\mathrm{y}$ arcilla en suelo $(\mathrm{r}=0.95 \mathrm{p}=0.003 ; \mathrm{r}=0.93$ $\mathrm{p}=0.007$; respectivamente). El contenido de fósforo durante el ciclo de estudio presentó diferencias significativas $(\mathrm{F}=1.13 \mathrm{p}<0.05 \mathrm{n}=$ 72) se registró el más alto contenido en enero $(0.38 \pm 0.04 \%$; $3861 \mathrm{mg} . \mathrm{Kg})$ y el menor en julio $(0.02 \pm 0.005 \%$; 209 mg.Kg). El contenido de fósforo total en los sitios de monitoreo no registró diferencias significativas $(\mathrm{F}=0.4$ $\mathrm{p}=0.8 \mathrm{n}=72)$, el máximo nivel lo presentó la Boca $(0.2 \pm 0.05 \%$; $2031 \mathrm{mg} . \mathrm{Kg})$ y el menor en Aspoquero (0.13 $\pm 0.02 \% ; 1260$ mg.Kg) (Fig. 4), se presentó correlación negativa con los niveles de $\mathrm{pH}$ en los sitios $(\mathrm{r}=-0.61 \mathrm{p}=0.004)$.

Degradación de hoja de mangle: Se registró un rápida pérdida de masa durante el primer mes desde $27.7 \%$ (5.54 $\pm 0.4 \mathrm{~g})$ de hoja de $R$. mangle; $30.2 \%(6.0 \pm 0.6 \mathrm{~g})$ de L. racemosa; $50.1 \%(10.9 \pm 0.2 \mathrm{~g})$ en hoja combinada y $51 \%(10.2 \pm 0.53 \mathrm{~g})$ para $A$. germinans. Entre especies, $A$. germinans registró la mayor degradación con $1.9 \pm 0.6 \mathrm{~g}$ en promedio de masa remanente al final del ciclo de estudio (365 días) (Fig. 5). El análisis ANCOVA no mostró diferencias significativas entre el porcentaje de degradación mensual de la hoja de $L$. racemosa y R. mangle $\left(\mathrm{F}=3.3 \mathrm{p}=0.08 \mathrm{r}^{2}=0.96\right)$, así como A. germinans y hojas combinadas $(\mathrm{F}=0.57 \mathrm{p}=$ $\left.0.46 r^{2}=0.98\right)$. Sin embargo, se identificaron diferencias entre $L$. racemosa y $R$. mangle con respecto a la especie A. germinans $(\mathrm{F}=319$ $\mathrm{p}<0.05 \mathrm{r}^{2}=0.98$ y $\mathrm{F}=363 \mathrm{p}<0.05 \mathrm{r}^{2}=0.98$ ); el análisis de medidas repetidas mostró datos similares de diferencias significativas entre la degradación mensual de hoja de $L$. racemosa y $R$. mangle ( $\mathrm{p}=0.08)$, así como, A. germinans el experimento de hojas combinadas $(\mathrm{p}=0.9)$, con registro de diferencias significativas entre el porcentaje de degradación mensual de $L$. racemosa y $R$. mangle con respecto a la especie A. germinans $(\mathrm{p}<0.05)$.

En el análisis por temporadas, la mayor degradación de hoja fue en nortes con una tasa (R) de degradación de $0.2 \pm 0.03$ g.día $^{-1} \mathrm{y}$ la menor en secas con $0.04 \pm 0.001$ g.día ${ }^{-1}$. Las especies $A$. germinans y el experimento de hoja

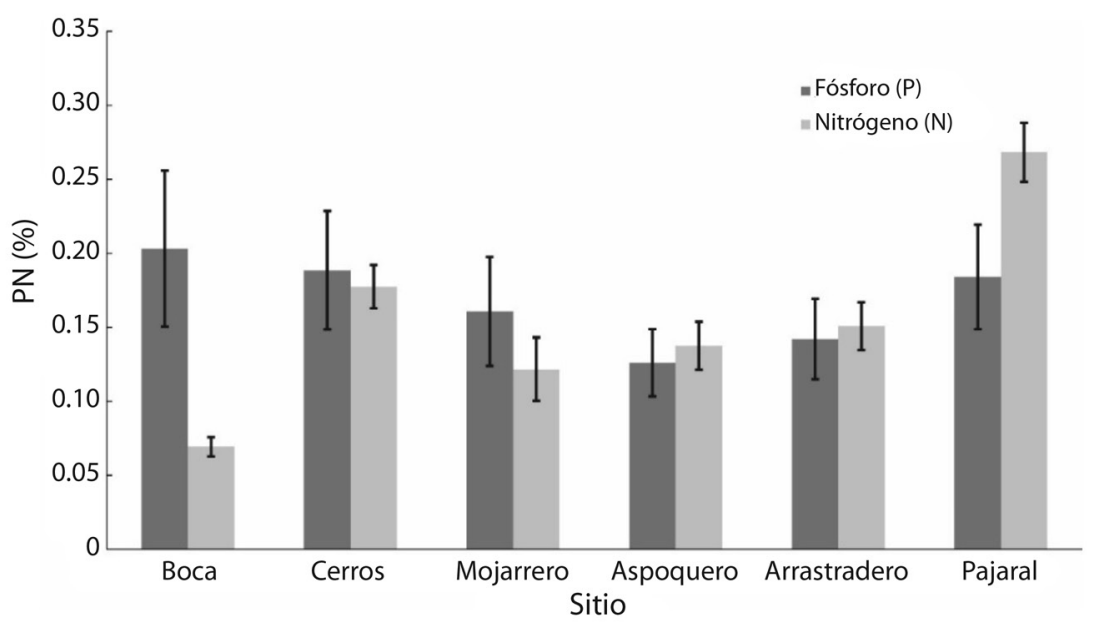

Fig. 4. Contenido de fósforo (PT) y nitrógeno (NT) total (\%) en la Laguna Mecoacán ( \pm ES).

Fig. 4. Phosphorus $(\mathrm{P})$ and nitrogen $(\mathrm{N})$ total content (\%) at Mecoacán Lagoon $( \pm \mathrm{SE})$. 


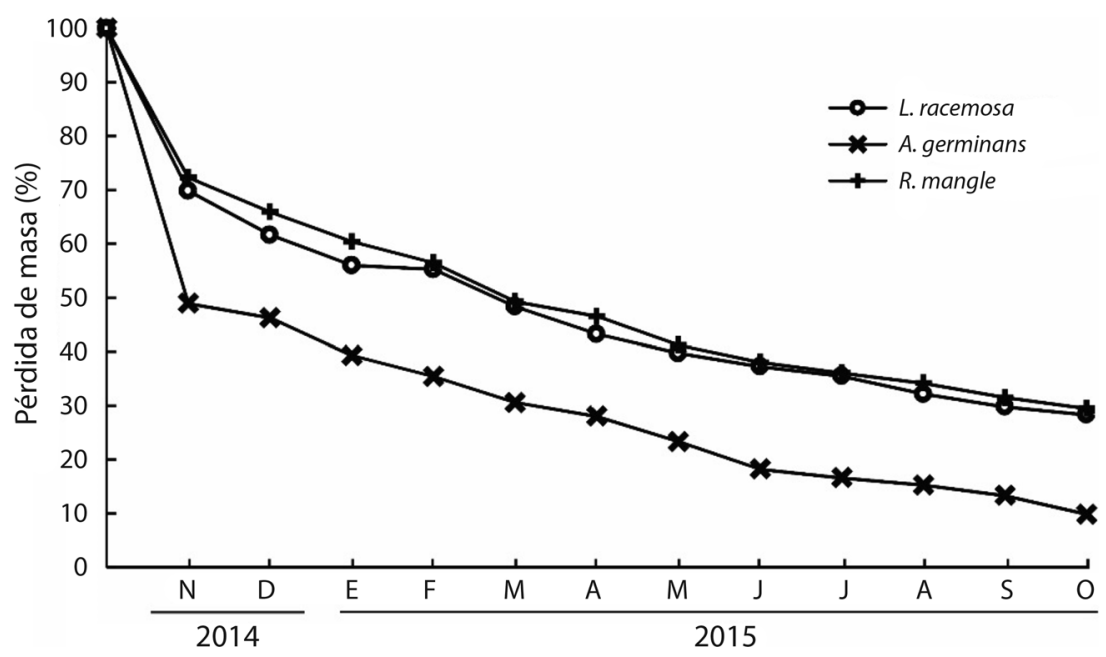

Fig. 5. Porcentaje de masa foliar remanente mensual para cada especie de manglar y hoja combinada $( \pm \mathrm{ES})$.

Fig. 5. Percentage of monthly leaf mass remaining for each species of mangrove and combined leaf ( \pm SE).

combinada no registraron diferencias significativas en la tasa de degradación (R) ( $p>0.05 n=$ 216). Sin embargo, se presentaron diferencias significativas en las especies $R$. mangle y $L$. racemosa $(\mathrm{p}<0.05 \mathrm{n}=216)$, con disminución de la tasa de degradación a partir de los 180 días (secas) $\left(0.03 \pm 0.003\right.$ y $0.04 \pm 0.001$ g.día $^{-1}$ respectivamente), las diferencias se conservan hasta finalizar el ciclo de monitoreo (Cuadro 1). Espacialmente, A. germinans y hoja combinada no presentaron diferencias significativas en la tasa de degradación $\mathrm{R}(\mathrm{p}>0.05 \mathrm{n}=216)$, mientras que las especies $R$. mangle $(\mathrm{F}=3.5$ $\mathrm{p}=0.004 \mathrm{n}=216)$ y L. racemosa $(\mathrm{p}<0.05 \mathrm{n}=$ 216) presentaron diferencias significativas, las diferencias se registraron en Mojarrero con el menor valor de $\mathrm{R}(0.05 \pm 0.006$ y $0.06 \pm 0.01$ g.día ${ }^{-1}$ para cada especie respectivamente) comparada con lo demás sitios (Fig. 6).

Las estimaciones del coeficiente de degradación diario $(\mathrm{k})$ en cada sitio, por especie y temporada se muestran en el cuadro 2 , se presentaron diferencias significativas entre sitios en la degradación de la hoja de $R$. mangle $(\mathrm{F}=12 \mathrm{p}<0.05 \mathrm{n}=216$ ), valores bajos de $\mathrm{k}$ para esta especie se presentaron en Mojarrero

\section{CUADRO 1}

Tasa de degradación (R) de masa foliar de las especies de manglar y hoja combinada por temporada

TABLE 1

Rate of degradation (R) of foliar mass of mangrove and combined leaf species and season

\begin{tabular}{lcccccc} 
& \multicolumn{2}{c}{ Nortes } & \multicolumn{2}{c}{ Secas } \\
\multicolumn{1}{r}{ Especie } & \multicolumn{2}{c}{ Días } & \multicolumn{2}{c}{ Lluvias } \\
& 60 & 120 & 180 & 240 & 300 & 365 \\
R. mangle & $0.15 \pm 0.02 \mathrm{a}$ & $0.1 \pm 0.01 \mathrm{a}$ & $0.06 \pm 0.004 \mathrm{~b}$ & $0.05 \pm 0.003 \mathrm{~b}$ & $0.05 \pm 0.002 \mathrm{~b}$ & $0.04 \pm 0.004 \mathrm{~b}$ \\
A. germinans & $0.25 \pm 0.04$ & $0.12 \pm 0.01$ & $0.09 \pm 0.004$ & $0.07 \pm 0.003$ & $0.06 \pm 0.002$ & $0.05 \pm 0.001$ \\
L. racemosa & $0.22 \pm 0.03 \mathrm{a}$ & $0.12 \pm 0.01 \mathrm{a}$ & $0.08 \pm 0.003 \mathrm{~b}$ & $0.07 \pm 0.003 \mathrm{~b}$ & $0.06 \pm 0.002 \mathrm{~b}$ & $0.05 \pm 0.001 \mathrm{~b}$ \\
Combinada & $0.15 \pm 0.04$ & $0.1 \pm 0.004$ & $0.06 \pm 0.003$ & $0.05 \pm 0.002$ & $0.04 \pm 0.001$ & $0.04 \pm 0.001$ \\
\hline
\end{tabular}

$( \pm \mathrm{ES})$, letras indican diferencias significativas entre días $(\mathrm{p}<0.05)$.

$( \pm \mathrm{SE})$, letters indicate significant differences between days $(\mathrm{p}<0.05)$. 


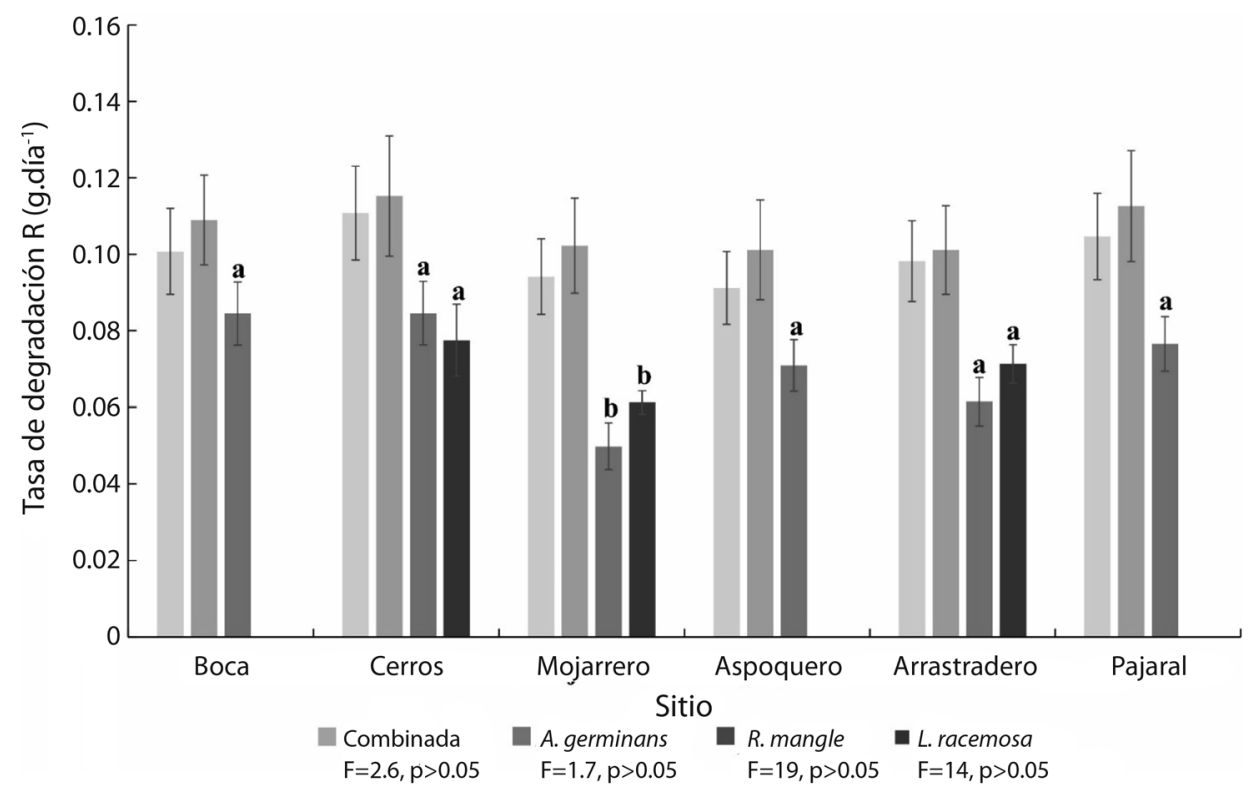

Fig. 6. Tasa de degradación (R) de masa foliar de las especies de manglar y hoja combinada por sitio de estudio, letras indican diferencias significativas entre sitios $(p<0.05)( \pm E S)$.

Fig. 6. Rate of degradation (R) of foliar mass of mangrove species and combined leaf per study site, letters indicate significant differences between sites $(p<0.05)( \pm S E)$.

\section{CUADRO 2}

Coeficiente de degradación $(\mathrm{k})$ por sitio y temporada de cada especie y hoja combinada de manglar

TABLE 2

Decay constant $(\mathrm{k})$ per site and season of each species and combined mangrove leaf

\begin{tabular}{|c|c|c|c|c|c|c|}
\hline & & \multicolumn{4}{|c|}{$\mathrm{k}$} & \multirow{2}{*}{$\mathrm{T}_{50}$} \\
\hline & & R. mangle & L. racemosa & A. germinans & Combinada & \\
\hline \multirow[t]{6}{*}{ Sitio } & Boca & $0.007 \pm 0.0005 \mathrm{a}$ & NA & $0.01 \pm 0.0006$ & $0.009 \pm 0.0007$ & 82 \\
\hline & Cerros & $0.006 \pm 0.0005 \mathrm{a}$ & $0.006 \pm 0.0004 \mathrm{a}$ & $0.01 \pm 0.001$ & $0.01 \pm 0.0008$ & 85 \\
\hline & Mojarrero & $0.003 \pm 0.0003 \mathrm{~b}$ & $0.004 \pm 0.0006 \mathrm{~b}$ & $0.008 \pm 0.0008$ & $0.008 \pm 0.0006$ & 121 \\
\hline & Aspoquero & $0.005 \pm 0.0004 \mathrm{a}$ & NA & $0.009 \pm 0.0008$ & $0.007 \pm 0.0005$ & 97 \\
\hline & Arrastradero & $0.004 \pm 0.0004 \mathrm{~b}$ & $0.005 \pm 0.0006 \mathrm{ab}$ & $0.008 \pm 0.0007$ & $0.008 \pm 0.0006$ & 107 \\
\hline & Pajaral & $0.006 \pm 0.0004 \mathrm{a}$ & NA & $0.01 \pm 0.0009$ & $0.009 \pm 0.0006$ & 84 \\
\hline \multirow[t]{3}{*}{ Temporada } & Nortes & $0.007 \pm 0.0004 \mathrm{a}$ & $0.006 \pm 0.0007 \mathrm{a}$ & $0.013 \pm 0.0008 \mathrm{a}$ & $0.012 \pm 0.0006 \mathrm{a}$ & 71 \\
\hline & Secas & $0.005 \pm 0.0002 b$ & $0.004 \pm 0.0003 \mathrm{~b}$ & $0.008 \pm 0.0002 \mathrm{~b}$ & $0.007 \pm 0.0002 \mathrm{~b}$ & 115 \\
\hline & Lluvias & $0.004 \pm 0.0002 \mathrm{~b}$ & $0.004 \pm 0.0002 \mathrm{~b}$ & $0.007 \pm 0.0002 \mathrm{~b}$ & $0.007 \pm 0.0002 \mathrm{~b}$ & 130 \\
\hline
\end{tabular}

$( \pm \mathrm{ES})$, letras indican diferencias significativas entre sitios y temporadas $(\mathrm{p}<0.05)$. (NA: no aplica).

$( \pm \mathrm{SE})$, letters indicate significant differences between sites and seasons $(\mathrm{p}<0.05)$. (NA: not aplicable).

$\mathrm{T}_{50}=$ tiempo estimado para degradación del $50 \%$ del material foliar.

$\mathrm{T}_{50}=$ estimated time for degradation of $50 \%$ of the leaf material.

$(0.003 \pm 0.0003)$ y Arrastradero $(0.004 \pm 0.0004)$, los rangos de variación para todas las especies fueron desde $0.003 \pm 0.0003$ a $0.01 \pm 0.001$, el valor más alto lo registró $A$. germinans
$(0.009 \pm 0.0003)$ y el experimento de hojas combinadas $(0.008 \pm 0.0002)$. Las especies que requieren mayor tiempo para degradación del $50 \%\left(\mathrm{~T}_{0.5}\right)$ de la hoja fueron $R$. mangle y 
L. racemosa (133 y 138 días respectivamente) mientras que $A$. germinans y combinada requieren menor tiempo (74 y 80 días respectivamente), en los sitios de monitoreo el menor tiempo se registró en Boca (82 días) y el mayor en Mojarrero (121 días) (Cuadro 2). Temporalmente, se presentaron diferencias significativas $(\mathrm{F}=21 \mathrm{p}<0.05 \mathrm{n}=216)$ con alta degradación (k) en nortes $(0.007 \pm 0.0004)$. Se identificó una alta correlación en $\mathrm{k}$ de las especies $R$. mangle y A. germinans con los valores de $\mathrm{pH}$ en los sitios de monitoreo $(\mathrm{r}=-0.85 \mathrm{p}=0.03$ y $\mathrm{r}=-0.7$ $\mathrm{p}=0.02$ respectivamente). Así mismo, se presentó correlación en $\mathrm{k}$ de $A$. germinans, y $R$. mangle con el contenido de fósforo $(\mathrm{r}=0.83 \mathrm{p}=$ 0.04 y r $=0.63 \mathrm{p}<0.05$ respectivamente). También se identificó una alta correlación positiva de $\mathrm{k}$ en $A$. germinans con el contenido de arcilla en suelo $(\mathrm{r}=0.6 \mathrm{p}=0.02)$. Además, los valores de materia orgánica y humedad en suelo se correlacionaron con $\mathrm{k}$ de $A$. germinans $(\mathrm{r}=0.65$ $\mathrm{p}<0.05$ y $\mathrm{r}=0.55 \mathrm{p}<0.05$ respectivamente).

\section{DISCUSIÓN}

La hipótesis sobre la diferente resistencia a la degradación de las hojas de las tres especies incluidas en este estudio (hipótesis 1 ) se confirmó, pues $R$. mangle y L. racemosa mostraron degradación más lenta en relación a la hoja de $A$. germinans. En este sentido, la mayor suculencia y más alto contenido de taninos de $R$. mangle (Lacerda et al., 1986) explica una descomposición más lenta; mientras que una baja cantidad de taninos y nitrógeno en $A$. germinans acelera el proceso de degradación (Middleton \& Mckee, 2001). Además, otros estudios han registrado degradación lenta para R. mangle y L. racemosa (Orihuela et al., 2004; Fernando \& Banner, 2009; Barroso-Matos et al., 2012) y un proceso más rápido para degradación de hoja del género Avicennia (Woodroffe, 1982; Wafar, Untawale \& Wafar, 1997; Middleton \& Mckee, 2001).

En la Laguna Mecoacán $R$. mangle y $L$. racemosa mostraron mayor resistencia a la degradación a partir de los 180 días (secas), además, $R$. mangle registró valores en secas y nortes similares a los reportados por Twilley, Lugo y Patterson-Zucca (1986) de 0.005 y 0.003 en los bosques de manglar en la cuenca sur de Florida, EU. Aké-Castillo et al. (2006) reportaron para la laguna Sontecomapan, Veracruz, valores de k para la especie $R$. mangle en temporada de Nortes $(0.0048 \pm 0.0005)$, secas $(0.0057 \pm 0.0004)$ y lluvias $(0.0084 \pm 0.0006)$, similares a la Laguna Mecoacán, a excepción de la temporada de lluvias con un valor menor casi en un $50 \%$.

El valor de k para $A$. germinans (0.01) fue similar al reportado por Sessegolo y Lana (1991) para Avicennia schaueriana Stapf \& Leachman (0.019) en la zona intermareal. La degradación más rápida de la hoja del género Avicennia es consistente con otros estudios de diversas áreas geográficas (Albright, 1976; Woodroffe, 1982; Wafar et al., 1997; Middleton \& Mckee, 2001). Los estudios de degradación de hoja del género Laguncularia son escasos. Por ejemplo, Menezes y Schaeffer-Novelli (2000) reportaron para Cananeia, Brasil, valores de $\mathrm{k}$ en L. racemosa (0.005) similares a los registrados en la Laguna Mecoacán $(0.005 \pm 0.0003)$.

En la hipótesis (2), sobre la esperada alta correlación positiva entre $\mathrm{k}$ y la mayor retención de humedad de los suelos arcilloso fue rechazada, ya que solamente se identificó correlación positiva de los valores de $\mathrm{k}$ en $A$. germinans con el contenido de arcilla. Sin embargo, se identificó alta correlación positiva del contenido de arcilla con el coeficiente de degradación de $A$. germinans y experimento de hojas combinadas. Así mismo, los sitios Pajaral y Cerros mostraron bajo valor de $\mathrm{T}_{0.5}$ con alto contenido de humedad que según Mackey y Smail (1996) promueve una mayor lixiviación y establece condiciones aptas para el deterioro por saprobiosis. Lugo y Snedaker (1974) sugirieron que la dinámica de descomposición en ambientes de manglar son influenciados principalmente por el contenido de humedad, $\mathrm{pH}$ del suelo, la aireación y la naturaleza del material de la planta (composición de la hoja).

En la Laguna Mecoacán, al predominar los suelos arenosos, en cuatro de seis sitios de 
muestreo (Boca, Cerros, Aspoquero y Arrastradero), las condiciones de retención de materia orgánica y humedad en suelo resultaron menores que en Mojarrero y Pajaral con mayor contenido de limo y arcilla, como fue documentado por De-Boer (2000) para una bahía intermareal en Mozambique. En cambio, en el sitio con mayor contenido de arcilla (Pajaral) se registró el más alto contenido de humedad, materia orgánica y nitrógeno, lo que concuerda con Bjorn y McClaugherty (2008), quienes mencionaron que los suelos con mayor contenido de arcilla son capaces de almacenar más materia orgánica que los suelos arenosos, así mismo propusieron que la textura es la propiedad física más importante del suelo e influye directamente en la dinámica del agua y nutrientes derivado de la porosidad y permeabilidad que presente el suelo.

La inmersión de la hojarasca en agua favorece la lixiviación de materiales lábiles (Robertson, 1988) y la actividad microbiana (Tam, Vrijmoed, \& Wong, 1990) que a su vez propicia una rápida degradación inicial. En la implementación del experimento de degradación en la Laguna Mecoacán (noviembre 2014) todos los sitios registraron inundación (excepto Arrastradero) (Fig. 3), lo que promovió los procesos de lixiviación de las hojas de mangle e incrementó los valores de k inicial. Así mismo, los coeficientes más altos de degradación en hoja de mangle se han asociado con las inundaciones de marea más frecuentes (Twilley, Lugo, \& Patterson-Zucca, 1986), como se identifica en el sitio Boca que se encuentra con mayor influencia marina (Fig. 1), mayor tiempo de inundación (Fig. 3) y el menor tiempo para la degradación del $50 \%\left(\mathrm{~T}_{0.5}\right)$ de la hoja con 82 días (Cuadro 2). Middleton y Mckee (2001) reportaron que en condiciones de inundación se promueve la lixiviación y se mantiene contenidos de humedad y temperatura en suelo propicias para la rápida degradación inicial.

En la Laguna Mecoacán se registró hasta $51 \%$ de degradación el primer mes en hoja de A. germinans. Davis, Coronado-Molina, Childers y Day-Jr. (2003) registraron que durante los primeros dos meses, el proceso de descomposición se puede atribuir a la lixiviación, acción fúngica y bacteriana. Aké-Castillo et al. (2006) agregaron que estos mecanismos son los responsables de una alta pérdida de masa foliar inicial de hasta el $50 \%$ en los manglares de la laguna Sontecomapan, Veracruz. Sierra-Rozo, Mancera y Santos-Martínez (2009) reportaron una alta degradación inicial en zonas de manglares de la isla San Andrés, Colombia, para A. germinans (54\%), R. mangle $(40 \%)$ y L. racemosa (36\%) y después del primer mes todas las especies tienden a disminuir la velocidad de degradación. La rápida degradación de hoja de mangle durante el primer mes es consistente con los resultados de otros estudios (Stela \& Salomão, 2009; Sánchez-Andrés, Sánchez-Carrillo, Alatorre, Cirujano, \& Álvarez-Cobelas, 2010; BarrosoMatos, Bernini, \& Rezende, 2012).

La dinámica de la materia orgánica está estrechamente relacionada a los ciclos biogeoquímicos del nitrógeno $(\mathrm{N})$ y fósforo $(\mathrm{P})$ en suelos de los humedales por los procesos de descomposición, mineralización y absorción de la vegetación (Chen \& Twilley, 1999), ya que estos elementos son requeridos por los organismos y actúan como nutrientes limitantes que pueden regular la productividad en los ecosistemas (Márquez, Senior, Martínez, \& González, 2007). La hipótesis (3) que menciona la alta correlación positiva del contenido de materia orgánica y nutrientes (fósforo y nitrógeno) en suelo del manglar con los coeficientes de degradación (k) de la hoja de mangle se rechazó, ya que solo se registró correlación positiva entre los valores de k en $A$. germinans con el contenido de materia orgánica. Sin embargo, la materia orgánica se correlacionó de forma positiva con el nitrógeno en suelo. El sitio con mayor contenido de arcilla (Pajaral), materia orgánica (Fig. 2) y nitrógeno (Fig. 4), registró la mayor productividad primaria promedio (base hojarasca) en comparación con los demás sitios durante el mismo ciclo de estudio con 50 g.m $\mathrm{m}^{-2} \cdot \mathrm{mes}^{-1}$ (datos tomados de Torres et al., 2017). En Ría Lagartos, México, se registraron valores similares de correlación $(\mathrm{r}=0.86)$ entre 
el contenido de materia orgánica y nitrógeno total en sedimento (Valdes \& Real, 2004).

En la parte este de la laguna (Pajaral) se registró la máxima acumulación de nitrógeno en sedimento (Fig. 4), la cual según AkéCastillo (2006) y Twilley et al., (1986) puede atribuirse a los aportes in situ en el proceso de degradación de la hoja de $R$. mangle por su alto contenido de nitrógeno en la hoja, que presentó las mayores densidades de árboles en Pajaral (1 350 org.ha $^{-1}$ ). Así mismo, Twilley et al., (1986) midieron bajo contenido de nitrógeno para $A$. germinans, que en la Laguna Mecoacán registró al oeste-noroeste de la laguna la mayor densidad de árboles de esta especie en Boca y Mojarrero (1820 y 3170 org.ha ${ }^{-1}$ respectivamente) y bajo contenido de nitrógeno en sedimento (Fig. 4). Los valores de densidad de árboles para $R$. mangle y $A$. germinans se tomaron de Torres et al., (2017) (medición de densidad de árboles en julio 2014).

El fósforo total (PT) es señalado por encima del nitrógeno como un limitante de la producción primaria en las zonas de manglares (Corredor, Howarth, Thilley, \& Morell, 1999). El PT de sedimento en la laguna se presentó en gradiente decreciente desde el mar (Boca) hacia la parte sur (Aspoquero) de la laguna
(Fig. 4), correlacionado de forma negativa con los valores de $\mathrm{pH}$ en los sitios de monitoreo, donde la condición de mayor de acidez aumentó el contenido de fósforo en sedimento. Un factor importante para la liberación del fósforo (P) es el pH, pues frecuentemente las condiciones reductivas del sedimento se mencionan como factor para aumentar el fósforo disponible (Oxmann, Schwendenmann, \& Lara, 2009). Además, el PT presentó correlación positiva con el coeficiente de degradación (k) de $A$. germinans y $R$. mangle, derivado de esto se puede atribuir una acumulación de fósforo en sedimento in situ, como ocurrió en los sitios Pajaral y Boca con los mayores coeficientes de degradación (k) y altos niveles de PT.

Los métodos de evaluación empleados para determinar la degradación de hoja de mangle presentan variaciones en su implementación, lo cual dificulta la comparación de los resultados (Sessegolo \& Lana, 1991) y fue corroborado al comparar los coeficientes de degradación $(\mathrm{k})$ y $\mathrm{T}_{50}$ para los géneros de mangle Rhizophora, Avicennia y Laguncularia (Cuadro 3) de diversos estudios en condiciones ambientales y aplicaciones metodológicas diferentes.

En conclusión, la velocidad de degradación de hoja para las tres especies de mangle

CUADRO 3

Coeficientes de degradación (k) y $\mathrm{T}_{50}$ de diferentes estudios de hoja de manglar

TABLE 3

Decay constant $(\mathrm{k})$ and $\mathrm{T}_{50}$ of different mangrove leaf studies

\begin{tabular}{|c|c|c|c|c|c|c|}
\hline Género & Ubicación & $\mathrm{k}$ & $r^{2}$ & $\mathrm{~T}_{50}$ & Referencia & $\begin{array}{c}\text { Condiciones de } \\
\text { experimento }\end{array}$ \\
\hline \multirow[t]{11}{*}{ Avicennia } & Cananeia (Brasil) & $1.2 \times 10^{-2}$ & 1 & 58 & Adaime (1985) & Sedimento \\
\hline & Florianopolis (Brasil) & $1.1 \times 10^{-3}$ & 0.94 & 30 & Panitz (1986) & Agua \\
\hline & Paranaguá (Brasil) & $1.9 \times 10^{-2}$ & - & - & Sessegolo y Lana (1991) & Sedimento \\
\hline & Kunduchi (Tanzania) & $4.5 \times 10^{-3}$ & 0.95 & 154 & Chale (1993) & Agua \\
\hline & Mandovi-Zuari (India) & $1.0 \times 10^{-2}$ & 0.96 & & Wafar, Untawale y Wafar (1997) & Agua \\
\hline & Tomago (Australia) & $8.6 \times 10^{-3}$ & 0.82 & - & Dick y Osunkoya (2000) & Sedimento \\
\hline & Maputo (Mozambique) & $4.0 \times 10-3$ (secas) & - & 171 & Stela y Salomao (2009) & Sedimento \\
\hline & & $1.4 \times 10-2$ (lluvias) & - & 48 & Stela y Salomao (2009) & Sedimento \\
\hline & $\begin{array}{l}\text { Golfo de California } \\
\text { (México) }\end{array}$ & $3.2 \times 10^{-2}$ & - & 5.8 & Sánchez-Andrés et al. (2010) & Sedimento \\
\hline & Río de Janeiro (Brasil) & $5.1 \times 10^{-3}$ & 0.75 & 138 & Barroso-Matos et al. (2012) & Sedimento \\
\hline & Golfo de México & $9.0 \times 10^{-3}$ & 0.94 & 74 & Éste estudio & Sedimento \\
\hline
\end{tabular}


CUADRO 3 (Continuación) / TABLE 3 (Continued)

\begin{tabular}{|c|c|c|c|c|c|c|}
\hline Género & Ubicación & $\mathrm{k}$ & $r^{2}$ & $\mathrm{~T}_{50}$ & Referencia & $\begin{array}{c}\text { Condiciones de } \\
\text { experimento }\end{array}$ \\
\hline \multirow[t]{16}{*}{ Rhizophora } & Cananeia (Brasil) & $8.8 \times 10^{-3}$ & 1 & 79 & Adaime (1985) & Sedimento \\
\hline & Florianopolis (Brasil) & $6.4 \times 10^{-3}$ & 0.91 & 90 & Panitz (1986) & Agua \\
\hline & Paranaguá (Brasil) & $6.0 \times 10^{-3}$ & - & - & Sessegolo y Lana (1991) & Sedimento \\
\hline & Mandovi-Zuari (India) & $2.4 \times 10^{-3}$ & 0.94 & - & Wafar, Untawale y Wafar (1997) & Agua \\
\hline & \multirow[t]{2}{*}{ Gazi Bay (Kenia) } & $5.7 \times 10^{-3}$ (secas) & - & - & Twilley et al. (1997) & Sedimento \\
\hline & & $1.1 \times 10^{-2}$ (lluvias) & - & - & Twilley et al. (1997) & Sediment0 \\
\hline & Matang (Malasia) & $1.6 \times 10^{-2}$ & 0.88 & 43 & Ashton, Hogarth y Ormond (1999) & Sedimento \\
\hline & Cananeia (Brasil) & $3.0 \times 10^{-3}$ & - & 189 & Menezes y Schaeffer-Novelli (2000) & Sedimento \\
\hline & \multirow[t]{2}{*}{ Maputo (Mozambique) } & $7.0 \times 10^{-3}$ (secas) & - & 98 & Stela y Salomao (2009) & Sedimento \\
\hline & & $2.8 \times 10^{-2}$ (lluvias) & - & 25 & Stela y Salomao (2009) & Sedimento \\
\hline & \multirow[t]{2}{*}{ Guayas (Ecuador) } & $1.8 \times 10^{-1}($ secas $)$ & 0.77 & 27 & Bosire et al. (2005) & Sedimento \\
\hline & & $4.1 \times 10^{-1}$ (lluvias) & 0.94 & 12 & Bosire et al. (2005) & Sedimento \\
\hline & \multirow[t]{2}{*}{ Golfo de México } & $5.7 \times 10^{-3}$ & 0.77 & 82 & Aké-Castillo et al. (2006) & Sedimento \\
\hline & & $8.4 \times 10^{-3}$ (lluvias) & 0.84 & 70 & Aké-Castillo et al. (2006) & Sedimento \\
\hline & Río de Janeiro (Brasil) & $2.7 \times 10^{-3}$ & 0.56 & 257 & Barroso-Matos et al. (2012) & Sedimento \\
\hline & Golfo de México & $5.2 \times 10^{-3}$ & 0.98 & 133 & Éste estudio & Sedimento \\
\hline \multirow[t]{8}{*}{ Laguncularia } & Cananeia (Brasil) & $7.5 \times 10^{-3}$ & 0.98 & - & Adaime (1985) & Sedimento \\
\hline & Florianopolis (Brasil) & $8.4 \times 10^{-3}$ & 0.95 & - & Panitz (1986) & Agua \\
\hline & Océano Pacífico (México) & $5.2 \times 10^{-2}$ & - & - & Flores-Verdugo et al. (1987) & Sedimento \\
\hline & Océano Pacífico (México) & $2.5 \times 10^{-1}$ & - & - & Flores-Verdugo et al. (1987) & Agua \\
\hline & Paranaguá (Brasil) & $1.2 \times 10^{-2}$ & - & - & Sessegolo y Lana (1991) & Sedimento \\
\hline & Cananeia (Brasil) & $5.0 \times 10^{-3}$ & - & - & Menezes y Schaeffer-Novelli (2000) & Sedimento \\
\hline & Río de Janeiro (Brasil) & $3.2 \times 10^{-3}$ & 0.73 & 216 & Barroso-Matos et al. (2012) & Sedimento \\
\hline & Golfo de México & $5.0 \times 10^{-3}$ & 0.95 & 138 & Éste estudio & Sedimento \\
\hline
\end{tabular}

(R. mangle, A. germinans y $L$. racemosa) depende de la composición de la hoja (suculencia), características físico químicas del suelo como la textura que promueve (arcilla) o limita (arena) la retención de humedad y el patrón de inundación (grado de exposición o inmersión en agua), además, el contenido de nutrientes en suelo está relacionado con la velocidad de degradación de la hoja de mangle (aporte de fósforo in situ) y la composición de especies que reciclan una mayor cantidad de nitrógeno (R. mangle). La estandarización experimental es imprescindible para mejorar las comparaciones de resultados de diferentes regiones, sobre todo con respecto a las escalas de tiempo, intervalos de recolectas, tamaño y porosidad de las bolsas de degradación, así como biomasa y tipos de hoja utilizada. Además se recomienda realizar estudios que permitan conocer el flujo y destino de la degradación de la hojarasca de manglar (nutrientes) en relación a la conectividad con hábitats adyacentes.

\section{AGRADECIMIENTOS}

Este trabajo fue desarrollado como parte de las actividades y con el apoyo de la Red Temática Áreas Naturales Protegidas CONACyT Proyecto 269540 y con el apoyo de la Red para el Conocimiento de los Recursos Costeros del Sureste de México.

\section{RESUMEN}

Los manglares son ecosistemas de importante productividad primaria, donde se establece un flujo de energía (nutrientes) con zonas adyacentes y su ambiente acuático, impulsado principalmente por los procesos de degradación. El objetivo del estudio fue estimar el coeficiente de degradación diario $(\mathrm{k})$ de hoja de mangle por medio de bolsas de 
degradación, en relación con los factores físico químicos del suelo y el aporte de nutrientes (fósforo y nitrógeno) en sedimento del manglar de la Laguna Mecoacán, Golfo de México. El coeficiente de degradación se estimó por medio de bolsas de degradación de hojarasca en seis sitios de monitoreo mensual. Se identificó una rápida degradación durante el primer mes de hasta $51 \%$ en Avicennia germinans (L.) Stearn asociado a procesos de lixiviación por condiciones de inundación. La degradación (k) de Rhizophora mangle $\mathrm{L}$. $(\mathrm{k}=0.0052 \pm 0.0002)(\mathrm{F}=12.2 \mathrm{p}<0.05$ $\mathrm{n}=216)$ y Laguncularia racemosa $(\mathrm{L}$.) Gaertn $(\mathrm{k}=0.005 \pm$ $0.0003)(\mathrm{F}=3.7 \mathrm{p}=0.02 \mathrm{n}=108)$ difieren significativamente de $A$. germinans $(\mathrm{k}=0.009 \pm 0.0003)(\mathrm{F}=1.2 \mathrm{p}=0.02$ $\mathrm{n}=216)$. En relación al $\mathrm{T}_{50}$ de $R$. mangle y L. racemosa presentaron mayor tiempo de degradación (133 y 138 días respectivamente) comparado con $A$. germinans (74 días). Se registró una correlación significativa entre la materia orgánica y la humedad del suelo con la constante de descomposición de $A$. germinans $(\mathrm{r}=0.65 \mathrm{p}<0.05$ y r $=0.55$ $\mathrm{p}<0.05$ respectivamente). El más alto contenido de nitrógeno total se dio en Pajaral (2 $683 \mathrm{mg} . \mathrm{Kg}$ ) y presentó alta correlación con el contenido de materia orgánica $(\mathrm{r}=0.9$ $\mathrm{p}=0.03$ ); en relación al fósforo total, el nivel más alto se presentó en Boca (2 $031 \mathrm{mg} . \mathrm{Kg}$ ) correlacionado de forma negativa con el $\mathrm{pH}(\mathrm{r}=-0.61 \mathrm{p}<0.05)$. En conclusión, las diferencias en la velocidad de degradación de las hojas de mangle dependen de la especie (composición foliar), tiempo de exposición o inmersión en agua (patrón de inundación) y heterogeneidad del sedimento (i.e., textura, $\mathrm{pH}$, contenido de humedad y densidad aparente).

Palabras clave: manglar; degradación; hojarasca; hidroperíodo; lixiviación.

\section{REFERENCIAS}

Adaime, R. R. (1985). Producción del bosque de manglar de Gamboa Nóbrega. (Tesis de Doctorado) Universidad de Sao Paulo, Brasil.

Aké-Castillo, J. A. (2006). Contribución y efecto de la hojarasca derivada de mangle en la productividad primaria y composición del fitoplancton de una laguna costera (Tesis de Doctorado). Instituto de Ecología A. C. Xalapa, Veracruz.

Aké-Castillo, J. A., Vázquez, G., \& López-Portillo, J. (2006). Litterfall and decomposition of Rhizophora mangle L. in a coastal lagoon in the southern Gulf of Mexico. Hydrobiologia, 559, 101-111.

Albright, L. J. (1976). In situ degradation of mangrove tissues. New Zealand Journal of Marine and Freshwater Research, 10, 385-389.

Ashton, E. C., Hogarth, P. J., \& Ormond, R. (1999). Breakdown of mangrove leaf litter in a managed mangrove forest in Peninsular Malaysia. Hydrobiología, 413, 77-88.
Bailey, G. W. (1968). Role of soils and sediment in water pollution control - Part 1: Reactions of Nitrogen and phosphorus compounds with soils and geological strata. Cornell University, New York: U. S. Department of the interior, Federal Water Pollution Control Administration, South East Water Laboratory.

Barba, E. M., Rangel, J., \& Ramos, R. (2006). Clasificación de los humedales de Tabasco mediante sistemas de información geográfica. Universidad y Ciencia, 22(002), 101-110.

Barroso-Matos, T., Bernini, E., \& Rezende, C. E. (2012). Decomposition of mangrove leaves in the estuary of Paraíba do Sul River Rio de Janeiro, Brazil. Latin American Journal of Aquatic Research, 40(2), 398-407.

Bjorn, B., \& McClaugherty, C. (2008). Plant Litter. Berlin Heidelberg, Germany: Springer.

Bosire, J. O., Dahdouh-Guebas, F., Kairo, J. G., Kazungu, J., Dehairs, F., \& Koedam, N. (2005). Litter degradation and $\mathrm{CN}$ dynamics in reforested mangrove plantations at Gazi Bay, Kenya. Biological Conservation, 126, 287-295.

Bouillon, S., Koedam, N., Raman, A. V., \& Dehairs, F. (2002). Primary producers sustaining macro-invertebrate communities in intertidal mangrove forests. Oecologia, 130, 441-448.

Chale, F. M. (1993). Degradation of mangrove leaf litter under aerobic conditions. Hydrobiología, 257, 177-183.

Chapman, S. B. (1986). Production Ecology and Nutrient Budgets. En P. D. Moore, \& S. B. Chapman, Methods in Plant Ecology (Second Edition, pp. 1-59). Oxford: Blackwell Scientific Publications.

Chen, R., \& Twilley, R. R. (1999). Pattern of mangrove forest structure and soil nutrient dynamics along the Shark River Estuary, Florida. Estuaries, 22, 955-970.

CONAGUA-SMN-EMAS. (2015). Estación Meteorológica Paraíso, Tabasco. Comisión Nacional del Agua, Servicio Meteorológico Nacional.

Corredor, J., Howarth, R., Thilley, R., \& Morell, J. (1999). Nitrogen Cycling and anthropogenic impact in the tropical interamerican seas. Biogeochemistry, 46, 163-178.

Davis, S. E., Coronado-Molina, D. L., Childers, D. L., \& Day-Jr, J. W. (2003). Temporally dependent C, N, and $\mathrm{P}$ dynamics associated with the decay of Rhizophora mangle L. leaf litter in oligotrophic mangrove wetlands of the Southern Everglades. Aquatic Botany, $75,199-215$.

De-Boer, W. F. (2000). Biomass dynamics of seagrasses and the role of mangrove and seagrass vegetation as different nutrient sources for an intertidal ecosystem. Aquatic Botany, 66(3), 225-239. 
Dick, T. M., \& Osunkoya, O. O. (2000). Influence of tidal restriction floodgates on decomposition of mangrove litter. Aquatic Botany, 63(3), 273-280.

Domínguez, J. C., Sánchez, A. J., Florido, R., \& Barba, E. (2003). Distribución de macrocrustáceos en Laguna Mecoacán, al sur del Golfo de México. Hidrobiología, 13, 127-136.

Domínguez-Domínguez, M., Zavala-Cruz, J., \& MartínezZurimendi, P. (2011). Manejo forestal sustentable de los manglares de Tabasco. Villahermosa, Tabasco, México: Secretaría de Recursos Naturales y Protección Ambiental, Colegio de Postgraduados.

Fernando, S. M., \& Banner, O. B. (2009). Litter fall and decomposition of mangrove species Avicennia marina and Rhizophora mucronata in Maputo Bay, Mozambique. Western Indian Ocean Journal of Marine Science, 8, 173-182.

Flores-Verdugo, F. J., Day-Jr, J. W., \& Briseño-Dueñas, R. (1987). Structure, litter fall, decomposition, and detritus dynamics of mangroves in a Mexican coastal lagoon with an ephemeral inlet. Marine Ecology Progress Series, 35, 83-90.

Gerritsen, J., \& Greening, H. S. (1989). Marsh seed banks of the Okefenokee swamp: effects of hydrologic regime and nutrients. Ecology, 70, 750-763.

Gómez, A. H. (1977). Determinación de corrientes en la laguna costera Mecoacán de Tabasco, México. Ciencias Marinas, 4(1), 67-80.

Harmon, M. E., Nadelhoffer, K. J., \& Blair, J. M. (1999). Measuring decomposition, nutrient turnover, and stores in plant litter. En G. P. Robertson, C. S. Bledsoe, D. C. Coleman, \& P. Sollins. Standard soil methods for long term ecological research (pp. 202-240). New York: Oxford Universitiy Press.

Hemminga, M. A., Slim, F. J., Kazunga, J., Ganssen, G. M., Nieuwenhuize, J., \& Kruyt, N. M. (1994). Carbon outwelling from a mangrove forest with adjacent sea grass beds and coral reefs (Gazi Bay, Kenya). Marine Ecology Progress Series, 106(3), 291-301.

Hernández, N. (2007). Abundancia y distribución de Cnidarios (Medusa) y Ctecnóforos (Agua Mala) En la Laguna Mecoacán Paraíso, Tabasco (Tesis de Licenciatura). Universidad Juárez Autónoma de Tabasco, Tabasco, México.

Infante, M. D. (2011). Estructura y dinámica de las selvas inundables de la planicie costera central del Golfo de México (Tesis de Doctorado). Instituto de Ecología A. C. Jalapa, Veracruz.

Infante-Mata, D., Tovilla-Hernández, C., Ovalle-Estrada, F., De-La-Presa, J. C., Cruz-Montes, G., \& LópezUrbina, J. H. (2014). Caracterización de la salinidad en la temporada de secas en manglares y otros humedales de la laguna Mecoacán, Tabasco. En M. González-Espinoza \& M. C. Brunel-Manse, Montañas, pueblo y agua. Dimensiones y realidades de la cuenca Grijalva (pp. 283-196). México D.F.: El Colegio de la Frontera Sur: Juan Pablos Editor.

Juman, R. A. (2005). Biomass, litterfall and decomposition rates for the fringed Rhizophora mangle forest lining the Bon Accord Lagoon, Tobago. Revista de Biología Tropical, 53(Suppl 1), 207-217.

Klute, A. (1986). Methods of soils analysis. Part 1. Physical and mineralogical methods (2nd ed.). Madison, Wisconsin, EUA: American Society of Agronomy, Inc. Soil Science Society of America, Inc. Publisher.

Lacerda, L. D., José, D. V., Rezende, C. E., Francisco, M. C., Wasserman, J. C., \& Martins, J. C. (1986). Leaf chemical characteristics affecting herbivory in a New World mangrove forest. Biotropica, 18(4), 350-355.

Lee, S. Y. (1990). Primary productivity and particulate organic matter flow in an estuarine mangrove-wetland in Hong Kong. Marine Biology, 106, 453-463.

Lee, S. Y. (1998). Ecological role of grapsid crabs in mangrove ecosystems: a review. Marine and Freshwater Research, 49, 335-343.

Lugo, A. E., \& Snedaker, S. C. (1974). The ecology of mangroves. Annual Review of Ecology and Systematic, 5, 39-64

Mackey, A. P., \& Smail, G. (1996). The Decomposition of Mangrove Litter in Subtropical Mangrove Forest. Hydrobiologia, 332, 93-98.

Márquez, A., Senior, W., Martínez, G., \& González, A. (2007). Concentraciones de nitrógeno y fósforo en sedimentos recientes de la laguna Los Patos, Estado Sucre, Venezuela. Boletín del Instituto Oceanográfico de Venezuela, 46(2), 137-145.

Melillo, J. M., Naiman, R. J., Aber, J. D., \& Eshleman, K. N. (1983). The influence of substrate quality and stream size on wood decomposition dynamics. Oecologia, 38, 281-285.

Menezes, G. V., \& Schaeffer-Novelli, Y. (2000). Producción y descomposición en bosques de manglar en la isla de Cardoso, Cananéia. Proceedings of the 15th Simpósio de ecossistemas brasileiros. ACIESP, Sao Paulo, 349-356.

Middleton, B. A., \& Mckee, K. L. (2001). Degradation of mangrove tissues and implications for peat formation in Belizean island forests. Journal of Ecology, 89, 818-828.

Moreno-Casasola, P., \& Warner, B. (2009). Breviario para describir, observar y manejar humedales. En Serie Costa Sustentable No. 1. Xalapa Ver. México: RAMSAR Instituto de Ecología A.C., CONANP, US Fish and Wildlife Service US State Department.

Neilson, M. J., Giddins, R. L., \& Richards, G. N. (1986). Effects of tannins on the palatibility of mangrove leaves in the tropical sesarmid crab Neosarmatium smithii (Crustacea: Decapoda: Sesarmidae). Marine Ecology Progress Series, 34, 185-186. 
OldeVenterink, H., Pieterse, N. M., Belgers, J. D., Wassen, M. J., \& de-Ruiter, P. C. (2002). N, P, and K budgets along nutrient availability and productivity gradients in wetlands. Ecological Applications, 12, 1010-1026.

Orihuela, D. C., Tovilla, H. F., Vester, H. F., \& Álvarez, T. (2004). Flujo de materia en un manglar de la costa de Chiapas, México. Madera y Bosques, 10(Es2, otoño), 45-61.

Oxmann, J. F., Schwendenmann, L., \& Lara, R. J. (2009). Interactions among phosphorus, $\mathrm{pH}$ and $\mathrm{Eh}$ in reforested mangroves, Vietnam: a three-dimensional spatial analysis. Biogeochemistry, 96,73-85.

Panitz, C. M. (1986). Produção e decomposição de serapilheira no mangue do rio Itacorubi, Ilha de Santa Catarina, Florianópolis, Brasil. São Carlos (Tesis de Doctorado). Universidad Federal de Sao Carlos, Brasil.

Pennington, T. D., \& Sarukhán, J. (2005). Árboles tropicales de México: manual para la identificación de las principales especies. México. D. F.: Universidad Nacional Autónoma de México/FCE.

Peralta, P. L., Infante, D. M., \& Moreno-Casasola, P. (2009). Construcción e instalación de Piezómetros. En P. Moreno-Casasola \& B. G. Warner, Breviario para describir, observar y manejar humedales (pp. 17-30). Xalapa, Veracruz, México: Serie Costa Sustentable No. 1. RAMSAR, Instituto de Ecología, A.C., CONANP, US Fish and Wildlife Service, US State Department.

Robertson, A. I. (1988). Decomposition of mangrove litter in tropical Australia. Journal of Experimental Marine Biology and Ecology, 166, 235-247.

Sánchez-Andrés, R., Sánchez-Carrillo, S., Alatorre, L. C., Cirujano, S., \& Álvarez-Cobelas, M. (2010). Litterfall dynamics and nutrient decomposition of arid mangroves in the Gulf of California: Their role sustaining ecosystem heterotrophy. Estuarine, Coastal and Shelf Science, 82(210), 191-199.

SEMARNAT, 2000. Norma Oficial Mexicana NOM021-SEMARNAT-2000. Ciudad de México, D. F., México: Diario Oficial de la Federación. http:// biblioteca.semarnat.gob.mx/janium/Documentos/ Ciga/libros2009/DO2280n.pdf

Sessegolo, G. C., \& Lana, P. C. (1991). Decomposition of Rhizophora mangle, Avicennia schaueriana and Laguncularia racemosa leaves in a mangrove of Paranaguá Bay (Southeastern Brazil). Botanica Marina, 34, 285-289.

Shunula, J. P., \& Whittck, A. (2001). A role of leaf litter degradation and nitrogen and carbon release in species from three families of mangroves in Zanzibar. En M. D. Richmond, \& J. Francis, Marine Science Development in Tanzania and Eastern Africa. Proceeding of the 20th Anniversary conference on Advances in Marine Science, Tanzania, 565.
Sierra-Rozo, O., Mancera, J. E., \& Santos-Martínez, A. (2009). Velocidad de descomposición de la hojarasca en diferentes sustratos de manglar durante la época de lluvias en San Andrés Isla, Caribe Colombiano. Boletin de Investigaciones Marinas y Costeras, $38(1), 59-84$.

Steel, A., \& Torrie, M. (1996). Bioestadística: Principios $y$ procedimientos (Segunda edición). México D. F. México: Edit. McGrawHill.

Steinke, T. D., Barnabas, A. D., \& Somaru, R. (1990). Structural changes and associated microbial activity accompanying decomposition of mangrove leaves in Mgeni Estuary. South African Journal Botany, 56(1), 39-48.

Stela, M. C., \& Salomão, O. B. (2009). Litter fall and decomposition of mangrove species Avicennia marina and Rhizophora mucronata in Maputo Bay, Mozambique. Western Indian Ocean Journal of Marine Sicence, 8(2), 173-182.

Tam, N. F., Vrijmoed, L. L., \& Wong, Y. S. (1990). Nutrient Dynamics Associated with Leaf Decomposition in a Small Subtropical Mangrove Community in Hong Kong. Bulletin of Marine Science, 47(1), 68-78.

Torres, J. R., Infante-Mata, D., Sánchez, A. J., EspinozaTenorio, A., \& Barba, E. (2017). Atributos estructurales, productividad (hojarasca) y fenología del manglar en la Laguna Mecoacán, Golfo de México. Revista de Biología Tropical, 65(4): 1592-1608.

Tovilla, C. (1994). Manglares. En E. G. De-La-Lanza \& M. Cáceres, Lagunas Costeras y el litoral mexicano (pp. 371-423). Universidad Autónoma de Baja California Sur, Ensenada.

Twilley, R. R., Lugo, A. E., \& Patterson-Zucca, C. (1986). Litter production and turnover in basin mangrove forest in southwest Florida. Ecology, 67(3), 670-683.

Twilley, R. R., Pozo, M., García, V. H., Rivera-Monroy, V. H., Zambrano, R., \& Bodero, A. (1997). Litter dynamics in riverine mangrove forest in the Guayas River estuary, Ecuador. Oecología, 111, 109-122.

Valdes, D. S., \& Real, E. (2004). Nitrogen and Phosphorus in water and sediments at Ría Lagartos coastal lagoon, Yucatan, Gulf of México. Indian Journal of Marine Sciences, 33(4), 338-345.

Wafar, S., Untawale, A. G., \& Wafar, M. (1997). Litter Fall and Energy Flux in a Mangrove Ecosystem. Estuari$n e$, Coastal and Shelf Science, 44, 111-124.

Walkley, A., \& Black, L. A. (1934). An examination of the method for determining soil organic matter, and proposed modification of the chromic acidtritation method. Soil Science, 37, 29-38.

Woodroffe, C. D. (1982). Litter production and decomposition in the New Zealand mangrove, Avicennia marina var. resinifera. New Zealand Journal of Marine and Freshwater Research, 16, 179-188. 